\title{
VORTICITY DYNAMICS AND NUMERICAL RESOLUTION OF NAVIER-STOKES EQUATIONS
}

\author{
Matania Ben-Artzi $^{1, *}$, Dalia Fishelov ${ }^{2}$ and Shlomo Trachtenberg ${ }^{3, \dagger}$
}

\begin{abstract}
We present a new methodology for the numerical resolution of the hydrodynamics of incompressible viscid newtonian fluids. It is based on the Navier-Stokes equations and we refer to it as the vorticity projection method. The method is robust enough to handle complex and convoluted configurations typical to the motion of biological structures in viscous fluids. Although the method is applicable to three dimensions, we address here in detail only the two dimensional case. We provide numerical data for some test cases to which we apply the computational scheme.
\end{abstract}

Mathematics Subject Classification. 35Q30, 65M06, 76D17.

Received: September 20, 2000. Revised: December 24, 2000.

\section{INTRODUCTION}

We present a new methodology for the numerical resolution of the dynamics of incompressible viscid Newtonian fluids. We focus here on the two-dimensional case. The method is applicable to the three-dimensional case as well, as we shall show in a forthcoming paper.

The underlying equations in this study are the Navier-Stokes equations $[8,23]$, which we recall below. In order to emphasize the new aspects in our approach, we restrict ourselves to the simplest case, namely, flow in a closed vessel, subject to "no slip" (zero velocity) boundary conditions. However, in some of our numerical examples, we use more general boundary conditions.

An important goal in our study is the calculation of motion of complex bodies in viscous fluids in a biological setting (see below). However, from the mathematical point-of-view, it means that the method should be sufficiently robust to handle rather convoluted or complex configurations.

So let $\Omega \subseteq R^{2}$ be a bounded domain with smooth boundary $\Gamma=\partial \Omega$.

Let $\mathbf{u}(\mathbf{x}, t)=\left(u^{1}(\mathbf{x}, t), u^{2}(\mathbf{x}, t)\right), \mathbf{x}=\left(x^{1}, x^{2}\right) \in \Omega$ be the velocity field for $t \geq 0$. The Navier-Stokes equations for incompressible viscid flow in $\Omega$ are

$$
\partial_{t} \mathbf{u}+(\mathbf{u} \cdot \nabla) \mathbf{u}=-\nabla p+\nu \Delta \mathbf{u}, \quad \nabla \cdot \mathbf{u}=\mathbf{0}
$$

\footnotetext{
* Partially supported by the Division for Research Funds, Ministry of Science, Israel.

$\dagger$ Partially supported by the US-Israel Binational Science Foundation and the Israel Academy of Sciences.

Keywords and phrases. Navier-Stokes equations, vorticity-streamfunction, numerical algorithm, vorticity boundary conditions.

1 Institute of Mathematics, The Hebrew University, Jerusalem 91904, Israel. e-mail: mbartzi@math.huji.ac.il

2 School of Mathematical Sciences, Tel Aviv University, Ramat Aviv, Tel Aviv 69978, Israel. e-mail: daliaf@math.tau.ac.il

3 Department of Membrane and Ultrastructure Research, The Hebrew University-Hadassah Medical School, P.O. Box 12271, Jerusalem 91120, Israel. e-mail: shlomot@cc.huji.ac.il
} 
where $p=p(\mathbf{x}, t)$ is the pressure and $\nu>0$ is the (constant) viscosity coefficient. Equations (1.1) are supplemented by giving the initial condition for velocity

$$
\mathbf{u}(\mathbf{x}, 0)=\mathbf{u}_{0}(\mathbf{x}), \mathbf{x} \in \Omega
$$

and a "no slip" boundary condition

$$
\mathbf{u}(\mathbf{x}, t)=\mathbf{0}, \mathbf{x} \in \Gamma \text { and all } t \geq 0 .
$$

It has become common practice to refer to $(\mathbf{u}, p)$ as "primitive variables". The most extensive theory concerning existence and uniqueness of solutions is formulated using these variables. This theory, originated by Leray [24] and Ladyzhenskaya [22] assumed (at least) that the total energy is finite $\left(i . e ., \int_{\Omega}|\mathbf{u}(\mathbf{x}, t)|^{2} \mathrm{~d} \mathbf{x}<\infty\right)$.

It proceeds to show the existence and uniqueness of a "continuously evolving" velocity field in this "energy space". We refer the reader to the books $[22,32]$ for comprehensive treatments of this theory, labeled as the "Galerkin Method" and to [32] for its numerical implementation.

Another approach to the numerical resolution of equations (1.1) is the so-called "projection methodology". Here the first equation is discretized to finite time intervals and the velocity field $\mathbf{u}(\mathbf{x}, t)$ is updated to the next time level as $\mathbf{u}(\mathbf{x}, t+\Delta t)$. This field is now projected onto the subspace of divergence-free fields, so as to satisfy the (discretized version of) the incompressibility condition $\underline{\nabla} \cdot \mathbf{u}=\mathbf{0}$. We refer to $[2,6,18,31]$ for detailed accounts.

One major drawback of the "primitive variables" approach lies in the difficulties encountered in the study of "vortical flows", i.e., flows involving vortices, vortex lines or areas of highly concentrated vorticity. From the mathematical point of view, the energy is then infinite [8], beyond the above-mentioned theoretical framework. From the numerical point of view, it becomes necessary to follow directly the evolution of the vorticity.

Needless to say, such vortical flows represent a large body of common phenomena in various scientific studies (meteorology, aeronautical science...). In particular, we aim at the hydrodynamic study of biological phenomena and structures spanning a wide range of dimensions, velocities, viscosities, and Reynolds numbers. Biological organisms, single or multicellular, are self-propelled and have dynamic, complex, and convoluted surfaces (boundaries). Propulsion (swimming) is carried out by whole body undulations or by means of specialized propellers capable of changing their geometries and physical properties. The analytical methods we propose here should be suitable to handle and analyze the hydrodynamics and microhydrodynamics of complex biological structures in their natural environments.

The "vorticity formulation", which lies at the basis of our present study, has been very extensively used in computational fluid dynamics, generating a panoply of so called "vortex methods". We refer the reader to the book [19] and the references cited therein. In the next section, we shall briefly touch further on some aspects associated with these methods.

Our approach is based on the "vorticity-streamfunction" formulation of the equations. It is closely related to the work of Dean-Glowinski-Pironneau [9], Quartapelle and coworkers [29,30] and W.E and J.-G. Liu [11-13]. See also $[26,33]$. The underlying philosophy of this method is to approximate the vorticity field by "sufficiently smooth" objects (see [3,4] for works justifying the "smoothing effect" in the vorticity equation). We then track its time evolution in terms of its associated streamfunction. Here the biharmonic operator plays a crucial role in the projection of the updated vorticity back onto the subspace where the "vorticity dynamics" takes place. Indeed, this is the vorticity analog of the divergence-free velocity fields. An important corollary of this approach is that we are able to avoid completely the difficulty of determining the vorticity on the boundary $\Gamma$. This means that "vorticity generation on the boundary" (a major problem encountered by standard methods) is automatically incorporated here. In fact, at each time step the no-slip condition is implemented via a modification of the whole vorticity field and not just its boundary values (as is the practice of all existing methods) .

In Section 2 we recall the vorticity-streamfunction formulation. We then introduce in detail our discretized scheme and the way it handles the boundary conditions. We compare our treatment with that suggested by other authors using the same formulation. Then in Section 3 we give some numerical examples. In the first 
two examples we measure the convergence rate of the scheme. Then, the time evolution of two vortices with opposite signs is represented. Numerical results are shown for a driven cavity, a double-driven cavity, a driven cavity with a solid small square inside the domain and a uniform flow over a square cylinder. Finally, we treat a backward facing step, where a recirculation zone behind the step is clearly captured.

\section{The Vorticity-PRojection MEthod}

The vorticity function, which is scalar in the two-dimensional case, is defined by

$$
\xi(\mathbf{x}, t)=\nabla \times \mathbf{u}=\partial_{x^{1}} u^{2}-\partial_{x^{2}} u^{1}, \mathbf{x} \in \Omega
$$

Taking the curl of the first equation in (1.1) we get

$$
\partial_{t} \xi+(\mathbf{u} \cdot \nabla) \xi=\nu \Delta \xi, \xi_{0}(\mathbf{x}, 0)=\nabla \times \mathbf{u}_{0}
$$

The velocity field $\mathbf{u}$ is obtained from $\xi$ by using (2.1) in conjunction with $\nabla \cdot \mathbf{u}=0$. This can be done via the "streamfunction formulation" [23] as follows. Due to $\nabla \cdot \mathbf{u}=0$, we have a function $\psi(\mathbf{x}, t)$ such that

$$
\mathbf{u}(\mathbf{x}, t)=\nabla^{\perp} \psi=\left(-\frac{\partial \psi}{\partial x^{2}}, \frac{\partial \psi}{\partial x^{1}}\right), \mathbf{x} \in \Omega, t \geq 0
$$

so that invoking $(2.1)$,

$$
\Delta \psi=\xi
$$

Thus, equations (2.2)-(2.4), along with the relation (2.3), serve as the "vorticity-streamfunction" formulation of the problem.

We note first that by integration of (2.1), the "no-slip" condition (1.3) implies

$$
\int_{\Omega} \xi(\mathbf{x}, t) \mathrm{d} \mathbf{x}=0, \text { all } t \geq 0
$$

Thus, for all $t, \xi(\bullet, t) \in L_{0}^{2}(\Omega)$, the $L^{2}$ functions of mean-value-zero. Furthermore, the relation $(2.3)$ and the "no slip" condition yield

$$
\psi(\mathbf{x}, t)=\frac{\partial}{\partial n} \psi(\mathbf{x}, t)=0, \mathbf{x} \in \Gamma, t \geq 0
$$

( $\frac{\partial}{\partial n}$ is the normal derivative). Note that (2.6) follows since $\psi=$ const. on the boundary and is only determined up to an additive constant. Using standard Hilbert space terminology [32], equation (2.6) can be restated as

$$
\psi(\bullet, t) \in H_{0}^{2}(\Omega)
$$

where $H_{0}^{2}(\Omega)$ is the closure of $C_{0}^{\infty}(\Omega)$ in the $H^{2}$ norm. Finally, from (2.4), we infer that

$$
\xi(\bullet, t) \in \Delta\left(H_{0}^{2}(\Omega)\right)=\text { the image of } H_{0}^{2}(\Omega) \text { under } \Delta \text {. }
$$

In particular, it is easily verified that $\Delta\left(H_{0}^{2}(\Omega)\right) \subseteq L_{0}^{2}(\Omega)$, so that (2.8) implies (2.5). Moreover, it can be shown [3] that $\Delta\left(H_{0}^{2}(\Omega)\right)$ is a closed subspace of $L_{0}^{2}(\Omega)$, so that

$$
L_{0}^{2}(\Omega)=\Delta\left(H_{0}^{2}(\Omega)\right) \oplus K_{0}(\Omega)
$$


where $\oplus$ denotes "direct sum" in Hilbert space and $K_{0}(\Omega)$ is the subspace of harmonic functions of mean-valuezero.

We note that $\Delta\left(H_{0}^{2}(\Omega)\right)$ is the subspace of "vorticity dynamics" (associated with the "no slip" boundary condition) and that equation (2.8) is the basis of our method.

When solving the system (2.2)-(2.4) we note that the streamfunction $\psi$ is subject to two (Dirichlet + Neumann) boundary conditions, thus rendering (2.4) as an overdetermined equation (for a given $\xi$ ). On the other hand, no (explicit) boundary conditions are provided for the vorticity, so that (2.2) is underdetermined. Thus, "the vorticity-streamfunction system is inextricably coupled" [17] p. 429. From both the mathematical and physical points of view the correct assignment of the vorticity on the boundary $\Gamma$ is crucial in obtaining accurate simulations of the flow. In particular, these boundary values determine the shape of the "boundary layer". In the context of "vortex methods" the most common treatment is that of deriving suitable boundary values for $\xi$ based on a simplified model, such as the "Prandtl model". We refer to $[7,8,14,19]$ for further details. In the context of the present vorticity-streamfunction formulation, the problem of "vorticity boundary conditions" has been studied by Anderson [1], Quartapelle et al. [10,29,30] and E and Liu [11-13]. Roughly speaking, the basic idea is to derive linear ("orthogonality") conditions that must be satisfied by the vorticity, as is seen from (2.9). This is a set of linear equations corresponding to the discrete analog of the subspace $K_{0}(\Omega)$ of harmonic functions. However, "unfortunately, this full system of equations has a rather cumbersome profile since the equations expressing the integral conditions have almost all coefficients different from zero" [10] p. 878. This is remedied and simplified in the method proposed by Dean, Glowinski and Pironneau [9] by splitting equations (2.2)-(2.4) into two separate Poisson equations. Another "localized" approach to the vorticity boundary conditions was presented by E and Liu [11-13].

To describe our approach for the discretization of (2.2)-(2.4), assume that a first approximation $\xi^{(1)}(\mathbf{x}, t+\Delta t)$ has been obtained from $\xi(\mathbf{x}, t)$ by using equation (2.2) (in discretized, explicit form). Then, we find the associated streamfunction $\psi(\mathbf{x}, t+\Delta t)$ by applying the Laplacian to $(2.4)$,

$$
\Delta^{2} \psi=\Delta \xi^{(1)}, \psi \in H_{0}^{2}(\Omega)
$$

Indeed, by (2.7), we seek $\psi \in H_{0}^{2}$, and standard elliptic theory gives that $\Delta^{2}$ is the natural (Dirichlet) operator for this boundary-value problem.

Finally, with $\psi$ defined by (2.10), we redefine

$$
\xi(\mathbf{x}, t+\Delta t)=\Delta \psi(\mathbf{x}, t+\Delta t)
$$

which is then used as the final value of the vorticity field at time $t+\Delta t$. Observe that this definition serves indeed as the projection of the first approximation $\xi^{(1)}(\mathbf{x}, t+\Delta t)$ on the subspace $\Delta\left(H_{0}^{2}(\Omega)\right)$. Here, not only the boundary values of $\xi^{(1)}$ are adjusted to conform with the "no slip" condition, but the whole vorticity field is suitably modified.

In practice, our algorithm consists of the following discretization of equations (2.2), (2.10) and (2.11). To simplify the exposition, we assume that $\Omega=[a, b] \times[c, d]$. We lay out a uniform grid

$$
\begin{aligned}
x_{i} & =a+i \Delta x, \quad y_{j} & =c+j \Delta y, \quad t_{n} & =n \Delta t, \\
\text { for } i & =0, \cdots, M, \quad j & =0, \cdots, K, \quad n & =0, \cdots, N,
\end{aligned}
$$

where $\Delta x=\frac{b-a}{M}, \Delta y=\frac{d-c}{K}$ and $\Delta t$ is determined by the stability considerations. 
Denote by $\xi_{i j}^{n}$ and $\psi_{i j}^{n}$ approximations for $\xi$ and $\psi$, respectively, at $\left(x_{i}, y_{j}, t_{n}\right)$.

For any discrete function $f_{i j}$, define

$$
\begin{aligned}
\delta_{x} f_{i j} & =\frac{f_{i+1, j}-f_{i-1, j}}{2 \Delta x} \\
\delta_{y} f_{i j} & =\frac{f_{i, j+1}-f_{i, j-1}}{2 \Delta y} \\
\delta_{x}^{2} f_{i j} & =\delta_{x}\left(\delta_{x} f_{i j}\right)=\frac{f_{i-1, j}-2 f_{i j}+f_{i+1, j}}{(\Delta x)^{2}} \\
\delta_{y}^{2} f_{i j} & =\delta_{y}\left(\delta_{y} f_{i j}\right)=\frac{f_{i, j-1}-2 f_{i j}+f_{i, j+1}}{(\Delta y)^{2}} .
\end{aligned}
$$

Given the discretized vorticity and streamfunction, $\xi_{i j}^{n}$ and $\psi_{i j}^{n}$, at time $t=n \Delta t$, we advance then to $t=$ $(n+1) \Delta t$ by the following algorithm:

(a) In the first step, $\xi$ is updated by a discretization of equation (2.2), i.e.,

$$
\bar{\xi}_{i j}^{n+1}=\xi_{i j}^{n}-\Delta t\left\{-\delta_{y} \psi_{i j}^{n} \cdot \delta_{x} \xi_{i j}^{n}+\delta_{x} \psi_{i j}^{n} \cdot \delta_{y} \xi_{i j}^{n}\right\}+\nu \Delta t\left\{\delta_{x}^{2} \xi_{i j}^{n}+\delta_{y}^{2} \xi_{i j}^{n}\right\}
$$

for all interior points $i=1, \cdots, M-1, j=1, \cdots, K-1$. On the boundary, $i=0, M$ or $j=0, N, \xi$ is left unchanged, i.e., $\bar{\xi}_{i j}^{n+1}=\xi_{i j}^{n}$.

Letting $\xi_{i j}^{n}=G^{n} \mathrm{e}^{i \alpha \Delta x} \mathrm{e}^{i \beta \Delta y}$, we have for the linear version of (2.12), that

$$
|G|^{2}=\left[1-\frac{4 \nu \Delta t}{(\Delta x)^{2}} \sin ^{2} \frac{\alpha \Delta x}{2}-\frac{4 \nu \Delta t}{(\Delta y)^{2}} \sin ^{2} \frac{\beta \Delta y}{2}\right]^{2}+\left[u \frac{\Delta t}{\Delta x} \sin (\alpha \Delta x)+v \frac{\Delta t}{\Delta y} \sin (\beta \Delta y)\right]^{2},
$$

where $u$ and $v$ are (frozen) constants.

If $\nu \leq 1$ the stability condition $|G| \leq 1+O(\Delta t)$ is clearly satisfied when

$$
\Delta t\left[\frac{1}{(\Delta x)^{2}}+\frac{1}{(\Delta y)^{2}}\right] \leq \frac{1}{2}
$$

(Observe that, in fact, if $\nu<<1$, we can replace the above condition by

$$
\Delta t\left[\frac{1}{(\Delta x)^{2}}+\frac{1}{(\Delta y)^{2}}\right] \leq \min \left(\frac{1}{2 \nu}, C\right),
$$

where $C>0$ can be chosen rather large).

We note here that with some modifications, the time integration can be converted to an implicit CrankNicholson scheme. Such a scheme will be presented later on in this section.

(b) In the second step of the algorithm, we construct $\psi$ from $\xi$ by discretizing (2.10), i.e., by

$$
\delta_{x}^{2} \delta_{x}^{2} \psi_{i, j}^{n+1}+2 \delta_{x}^{2} \delta_{y}^{2} \psi_{i, j}^{n+1}+\delta_{y}^{2} \delta_{y}^{2} \psi_{i, j}^{n+1}=\delta_{x}^{2} \bar{\xi}_{i, j}^{n+1}+\delta_{y}^{2} \bar{\xi}_{i, j}^{n+1}
$$

for all interior points $i=2, \cdots, M-2, j=2, \cdots, K-2$, i.e., for all points excluding boundary points and nearboundary points. On the boundary points $i=0, M$ or $j=0, K, \psi=0$ is applied, and on near-boundary points $i=1, M-1$ or $j=1, K-1, \frac{\partial \psi}{\partial n}$ is approximately imposed. For example, for $i=1 \psi(1, j)=\psi(0, j)+\Delta x \frac{\partial \psi}{\partial x}(0, j)$, where $\frac{\partial \psi}{\partial x}(0, j)$ is known from the boundary condition (2.6). Note that the left hand side of (2.13) can be computed for $i=2, \cdots, M-2, j=2, \cdots, K-2$ from the values of $\psi_{i, j}^{n}$ at $i=0, \cdots, M, j=0, \cdots, K$, and the right hand side of (2.13) can be computed for $i=2, \cdots, M-2, j=2, \cdots, K-2$ from the values of $\bar{\xi}_{i, j}^{n+1}$ at 
$i=1, \cdots, M-1, j=1, \cdots, K-1$, i.e., vorticity values at interior points (excluding boundary points $i=0, M$ or $j=0, K)$.

The resulting set of equations is of the form $A \Psi=B$, where $\Psi$ is an unknown vector of the discrete streamfunction values at interior points and $A$ is a symmetric banded matrix. The latter may be solved by a Cholesky factorization of $A$ into $L L^{T}$, where $L$ is a banded lower triangular matrix. In [5] Bjorstad improved the latter by decomposing $A$ into matrices that represent discrete operators in each spatial direction. The resulting algorithm has a complexity of $O(M K)$.

(c) Finally, we redefine $\xi$ at $t=(n+1) \Delta t$ by a discrete version of the projection described in equation (2.11), i.e., by

$$
\xi_{i, j}^{n+1}=\delta_{x}^{2} \psi_{i, j}^{n+1}+\delta_{y}^{2} \psi_{i, j}^{n+1}
$$

for all points $i=0, \cdots, M, j=0, \cdots, K$. The boundary values are determined by one-sided differentiations.

Thus, equations (2.12)-(2.14) determine the discrete values of $\xi$ and $\psi$ at $t=(n+1) \Delta t$.

One can also construct an implicit second order scheme (Crank-Nicholson) as follows.

$$
\frac{\bar{\xi}_{i j}^{n+1}-\xi_{i j}^{n}}{\Delta t}=-\left\{-\delta_{y} \psi_{i j}^{n+1 / 2} \cdot \delta_{x} \xi_{i j}^{n+1 / 2}+\delta_{x} \psi_{i j}^{n+1 / 2} \cdot \delta_{y} \xi_{i j}^{n+1 / 2}\right\}+\frac{\nu}{2}\left\{\delta_{x}^{2} \xi_{i j}^{n}+\delta_{y}^{2} \xi_{i j}^{n}+\delta_{x}^{2} \xi_{i j}^{n+1}+\delta_{y}^{2} \xi_{i j}^{n+1}\right\}
$$

Here $\xi_{i j}^{n+1 / 2}$ is obtained by Taylor expansion of $\xi$, using the differential equation (2.2), i.e.,

$$
\begin{aligned}
\xi\left(x_{i}, y_{j}, t+\Delta t / 2\right) & =\xi\left(x_{i}, y_{j}, t\right)+\frac{\Delta t}{2} \xi_{t}\left(x_{i}, y_{j}, t\right)+O\left(\Delta t^{2}\right) \\
& =\xi\left(x_{i}, y_{j}, t\right)+\frac{\Delta t}{2}\left\{-\left(\mathbf{u}\left(x_{i}, y_{j}, t\right) \cdot \nabla\right) \xi\left(x_{i}, y_{j}, t\right)+\nu \Delta \xi\left(x_{i}, y_{j}, t\right)\right\}+O\left(\Delta t^{2}\right) .
\end{aligned}
$$

Therefore,

$$
\bar{\xi}_{i j}^{n+1 / 2}=\xi_{i j}^{n}-\frac{\Delta t}{2}\left\{-\delta_{y} \psi_{i j}^{n} \cdot \delta_{x} \xi_{i j}^{n}+\delta_{x} \psi_{i j}^{n} \cdot \delta_{y} \xi_{i j}^{n}\right\}+\frac{\nu \Delta t}{2}\left\{\delta_{x}^{2} \xi_{i j}^{n}+\delta_{y}^{2} \xi_{i j}^{n}\right\}
$$

Then we construct $\psi_{i j}^{n+1 / 2}$ via step (b), i.e.,

$$
\delta_{x}^{2} \delta_{x}^{2} \psi_{i, j}^{n+1 / 2}+2 \delta_{x}^{2} \delta_{y}^{2} \psi_{i, j}^{n+1 / 2}+\delta_{y}^{2} \delta_{y}^{2} \psi_{i, j}^{n+1 / 2}=\delta_{x}^{2} \bar{\xi}_{i, j}^{n+1 / 2}+\delta_{y}^{2} \bar{\xi}_{i, j}^{n+1 / 2}
$$

for all interior points $i=2, \cdots, M-2, j=2, \cdots, K-2$. On the boundary and near-boundary points - the boundary conditions are imposed on $\psi$ in the same way as in step (b) above.

Finally, $\xi_{i j}^{n+1 / 2}$ is updated by step (c) as follows.

$$
\xi_{i, j}^{n+1 / 2}=\delta_{x}^{2} \psi_{i, j}^{n+1 / 2}+\delta_{y}^{2} \psi_{i, j}^{n+1 / 2}
$$

for all points $i=0, \cdots, M, j=0, \cdots, K$.

Now, the approximated values of $\xi_{i j}^{n+1 / 2}$ and $\psi_{i j}^{n+1 / 2}$ are substituted in (2.15), which yields an implicit second order scheme for (2.2). From here, we proceed with steps (b) and (c) as before.

The stability condition for this scheme, based on linear analysis, is

$$
\Delta t\left[\frac{\max |u|}{\Delta x}+\frac{\max |v|}{\Delta y}\right] \leq 1
$$




\section{Numerical RESUltS}

We present here several problems, for which we applied our scheme. All the examples were studied using the explicit scheme. In the first and second examples we measure the rate of convergence.

The first test problem has the following initial vorticity

$$
\xi(x, y, 0)=\pi\left(\cos (2 \pi x) \sin ^{2}(\pi y)+\sin ^{2}(\pi x) \cos (2 \pi y)\right),
$$

and streamfunction

$$
\psi(x, y, 0)=(1 / \pi) \sin ^{2}(\pi x) \sin ^{2}(\pi y),
$$

for $0 \leq x, y \leq 1[2]$. The appropriate boundary conditions are $\psi=\frac{\partial \psi}{\partial n}=0$ on $\Gamma$.

We have measured the numerical rate of convergence for $\nu=0.01,0.001,0.0001$. For each $\nu$ we computed the solution on a uniform grid with $h=\Delta x=\Delta y=1 / 2^{n}$, for $n=4,5,6,7$ to $t=0.1$. To estimate the rate of convergence, we compare the streamfunction, obtained on a grid with mesh size $h, \psi_{h}$, with the one obtained on the next finer grid, $\psi_{h / 2}$.

This is done by computing $e(h)=\left\|\psi_{h / 2}^{\text {ave }}-\psi_{h}\right\|_{l^{2}}$. Here $\psi_{h / 2}^{\text {ave }}$ is the averaged value of $\psi_{h / 2}$, computed at the coarser mesh points, and the average is taken on the coarse-point itself and its next four finer-mesh neighbors. $l^{2}$ indicates the discrete $L^{2}$ norm. The results are represented in Table 1. Note that the rate of convergence is then given by

$$
p=\log _{2}\left[\frac{e(h)}{e(h / 2)}\right]
$$

TABLE 1.

\begin{tabular}{|l|c|c|c|c|c|}
\hline \multicolumn{1}{|c|}{ mesh } & $16-32$ & Rate & $32-64$ & Rate & $64-128$ \\
\hline$\nu=0.01$ & $1.167 \times 10^{-3}$ & 2.40 & $3.162 \times 10^{-4}$ & 2.31 & $6.379 \times 10^{-5}$ \\
\hline$\nu=0.001$ & $2.031 \times 10^{-3}$ & 2.06 & $4.842 \times 10^{-4}$ & 2.12 & $1.115 \times 10^{-4}$ \\
\hline$\nu=0.0001$ & $2.066 \times 10^{-3}$ & 2.02 & $5.063 \times 10^{-4}$ & 2.04 & $1.229 \times 10^{-4}$ \\
\hline
\end{tabular}

The computed results indicate that the numerical rate of convergence is approximately 2 .

A second test problem has a known exact solution [6]

$$
\xi(x, y, t)=\mathrm{e}^{-2 t} \sin x \sin y, 0 \leq x, y \leq \pi .
$$

Table 2 displays the error $e$ and the relative error $e_{\mathrm{r}}$, where

$$
e=\left\|\xi_{\text {comp }}-\xi_{\text {exact }}\right\|_{l^{2}},
$$

and $e_{\mathrm{r}}=e /\left\|\xi_{\text {exact }}\right\|_{l^{2}}$. Here, $\xi_{\text {comp }}$ and $\xi_{\text {exact }}$ are computed and exact vorticities, respectively. We present results for different time-levels and number of mesh points, with $\nu=1$.

The third test problem, on which we have implemented the scheme, contains two concentrated vortices of opposite signs, located at two different points of the domain $\Omega=[-5,5] \times[-5,5]$. Thus

$$
\xi(x, y, 0)= \begin{cases}\left\{1-64\left[(x-2)^{2}+(y-2)^{2}\right]\right\}^{7}, & (x-2)^{2}+(y-2)^{2} \leq 1 / 64, \\ -\left\{1-64\left[(x+2)^{2}+(y+2)^{2}\right]\right\}^{7}, & (x+2)^{2}+(y+2)^{2} \leq 1 / 64 \\ 0, & \text { otherwise }\end{cases}
$$

Figures $1 \mathrm{a}-\mathrm{d}$ represent the calculated vorticity at $t=0.0078,0.234,0.391,7.812$, respectively, with a spatial mesh of $41 \times 41$ points. Similarly, Figures $2 \mathrm{a}-\mathrm{d}$ show the time evolution of the streamfunction. Note that at the last step the vorticity and the streamfunction decay to the order of $10^{-6}$. 
TABLE 2 .

\begin{tabular}{|r|l|l|l|l|l|}
\hline \multicolumn{1}{|c|}{ mesh } & $\mathrm{M}=\mathrm{K}=10$ & Rate & $\mathrm{M}=\mathrm{K}=20$ & Rate & $\mathrm{M}=\mathrm{K}=40$ \\
\hline$t=1 e$ & $2.522 \times 10^{-3}$ & 2.67 & $3.943 \times 10^{-4}$ & 2.00 & $9.861 \times 10^{-5}$ \\
$e_{\mathrm{r}}$ & $1.151 \times 10^{-2}$ & & $1.849 \times 10^{-3}$ & & $4.630 \times 10^{-4}$ \\
\hline$t=2 e$ & $3.334 \times 10^{-3}$ & 2.64 & $5.344 \times 10^{-5}$ & 2.67 & $1.332 \times 10^{-5}$ \\
$e_{\mathrm{r}}$ & $1.152 \times 10^{-2}$ & & $1.849 \times 10^{-3}$ & & $4.630 \times 10^{-4}$ \\
\hline$t=3 e$ & $4.632 \times 10^{-5}$ & 2.67 & $7.242 \times 10^{-6}$ & 2.00 & $1.806 \times 10^{-6}$ \\
$e_{\mathrm{r}}$ & $1.152 \times 10^{-2}$ & & $1.849 \times 10^{-3}$ & & $4.630 \times 10^{-4}$ \\
\hline$t=4 e$ & $6.124 \times 10^{-6}$ & 2.64 & $9.815 \times 10^{-7}$ & 2.64 & $2.477 \times 10^{-7}$ \\
$e_{\mathrm{r}}$ & $1.152 \times 10^{-2}$ & & $1.849 \times 10^{-3}$ & & $4.630 \times 10^{-4}$ \\
\hline
\end{tabular}

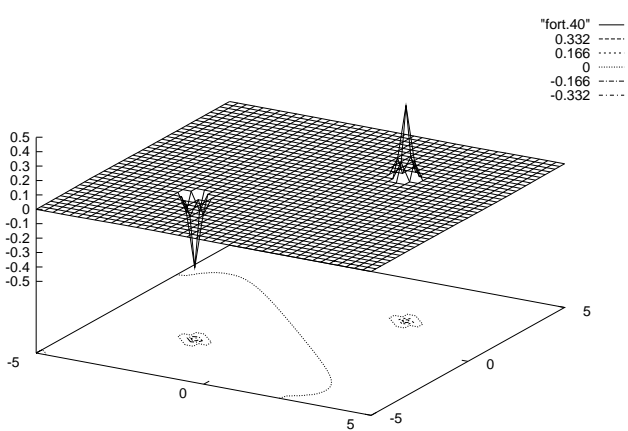

(a) $t=0.0078$

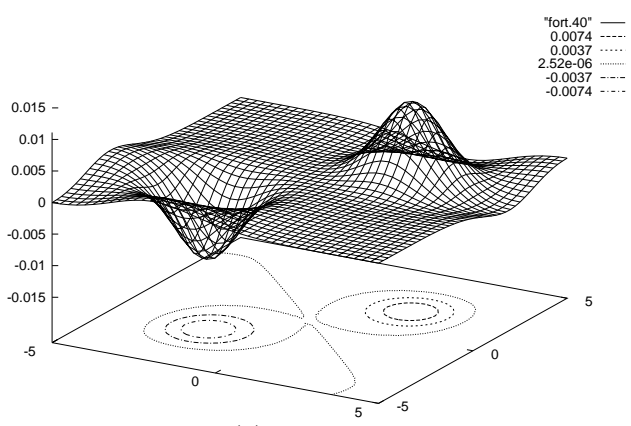

(c) $t=0.391$

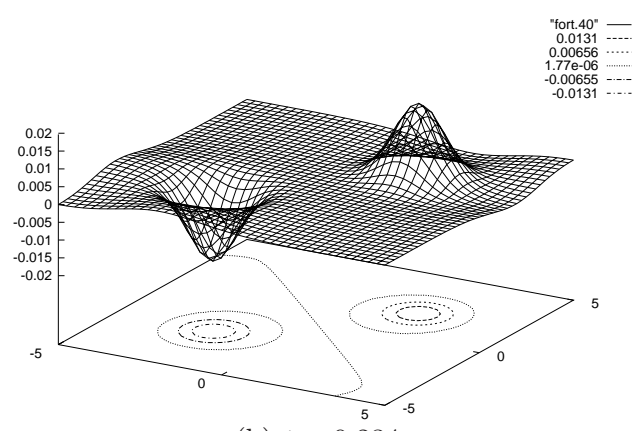

(b) $t=0.234$

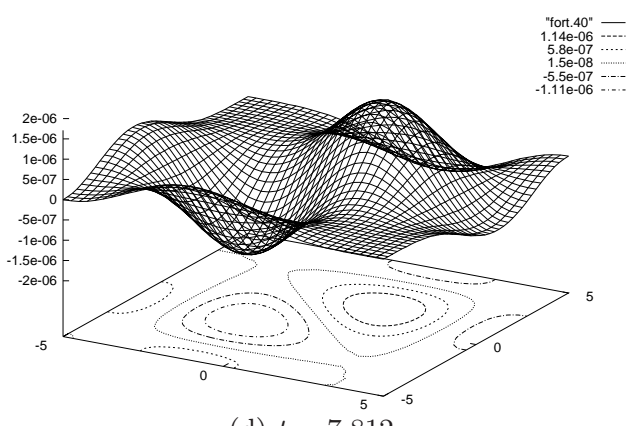

(d) $t=7.812$

FIGURE 1. Vorticity contours for two opposite-sign vortices.

Next, we show numerical results for a driven cavity with $\nu=1 / 400$. Here the domain is $\Omega=[0,1] \times[0,1]$ and the fluid is driven in the $x$-direction on the top section of the boundary $(y=1)$. Thus, $u=1, v=0$ for $y=1$, and $u=v=0$ for $x=0, x=1$ and $y=0$. In Table 3 we present computational quantities for different meshes and time-levels. We show $\max |\psi|,(\bar{x}, \bar{y})$, where $(\bar{x}, \bar{y})$ is the point where $\max |\psi|$ occurs, and the value of the vorticity at these points. The meshes are of $129 \times 129,193 \times 193$ and $257 \times 257$ points and the time levels are $t=9,15,30,45$. Note that the highest absolute value of the streamfunction at the latest time step is 0.1128 . Here the maximum occurs at $(\bar{x}, \bar{y})=(0.5664,0.5781)$, where the value of the vorticity is 2.3185 . Note 
TABLE 3.

\begin{tabular}{|c|c|c|c|c|}
\hline time & quantity & $129 \times 129$ & $193 \times 193$ & $257 \times 257$ \\
\hline 9 & $\max |\psi|$ & 0.1224 & 0.1177 & 0.1151 \\
& $(\bar{x}, \bar{y})$ & $(0.5703,0.5859)$ & $(0.5729,0.5885)$ & $(0.5742,0.5859)$ \\
& $\xi(\bar{x}, \bar{y})$ & 2.8493 & 2.7416 & 2.6879 \\
\hline 15 & $\max |\psi|$ & 0.1223 & 0.1169 & 0.1139 \\
& $(\bar{x}, \bar{y})$ & $(0.5625,0.5859)$ & $(0.5677,0.5803)$ & $(0.5664,0.5781)$ \\
& $\xi(\bar{x}, \bar{y})$ & 2.6083 & 2.4630 & 2.3964 \\
\hline 30 & $\max |\psi|$ & 0.1217 & 0.1159 & 0.1128 \\
& $(\bar{x}, \bar{y})$ & $(0.5625,0.5859)$ & $(0.5625,0.5781)$ & $(0.5664,0.5781)$ \\
& $\xi(\bar{x}, \bar{y})$ & 2.5520 & 2.4016 & 2.3196 \\
\hline 45 & $\max |\psi|$ & 0.1214 & 0.1159 & 0.1128 \\
& $(\bar{x}, \bar{y})$ & $(0.5625,0.5859)$ & $(0.5625,0.5781)$ & $(0.5664,0.5781)$ \\
& $\xi(\bar{x}, \bar{y})$ & 2.5460 & 2.4003 & 2.3185 \\
\hline
\end{tabular}

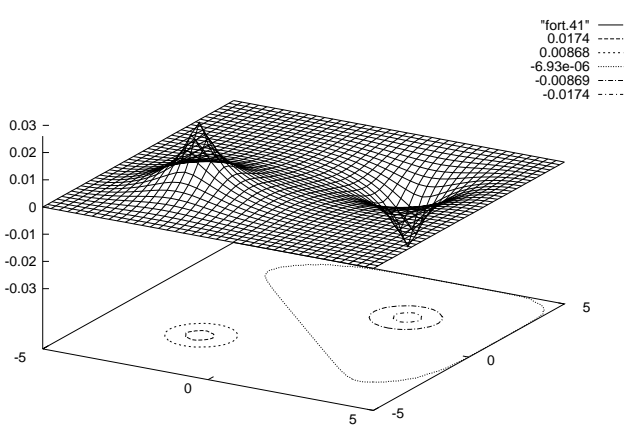

(a) $t=0.0078$

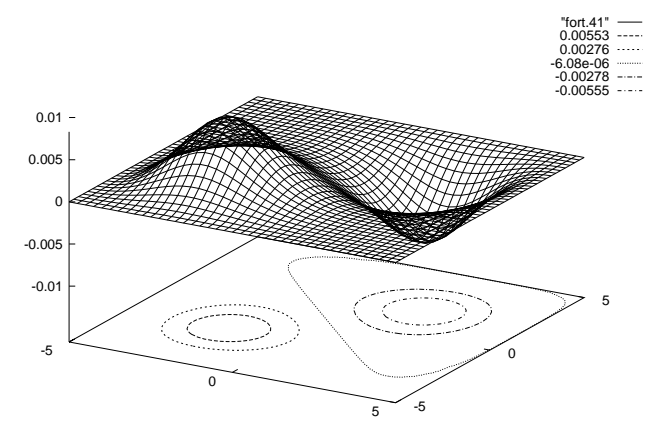

(c) $t=0.391$

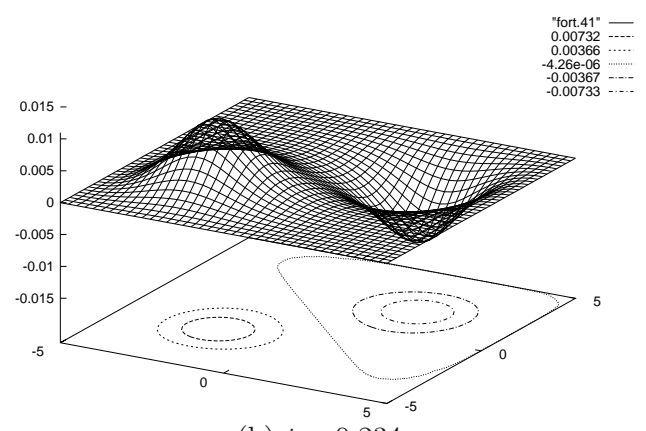

(b) $t=0.234$

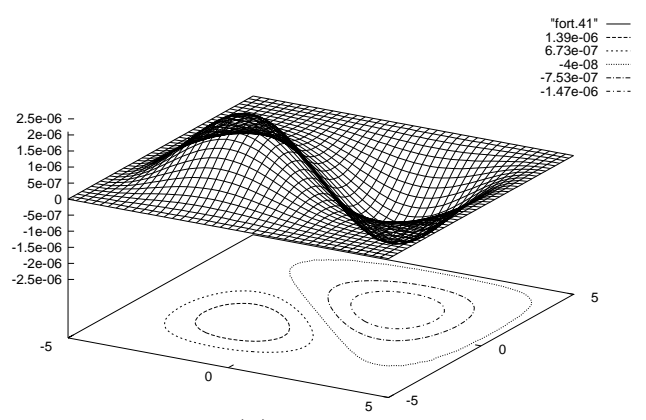

(d) $t=7.812$

FIGURE 2. Streamfunction contours for two opposite-sign vortices.

also that the location $(\bar{x}, \bar{y})$ for the finest grid has been stabilized at $t=15$. In [15] $\max |\psi|=0.1139$ occurs at $(0.5547,0.6055)$, where the value of the vorticity is 2.2947 .

In Table 4 we display the same flow quantities as in Table 3 , but for $\nu=1 / 3200$ at $t=60,120,150,180$. The meshes are of $193 \times 193,257 \times 257$ and $513 \times 513$ points. Note that with each of the meshes the flow quantities tend to converge to a steady state as time progresses. At the latest time level on the finest grid the maximal value of $|\psi|$ is 0.1246 , which is obtained at $(\bar{x}, \bar{y})=(0.5254,0.5078)$, and $\xi(\bar{x}, \bar{y})=2.0447$. The values reported in $[15]$ are $\max |\psi|=0.1204$, obtained at $(0.5165,0.5469)$, with $\xi=1.9886$. Figures $3 \mathrm{a}-\mathrm{d}$ and $4 \mathrm{a}-\mathrm{d}$ display the 




(a) $t=60$

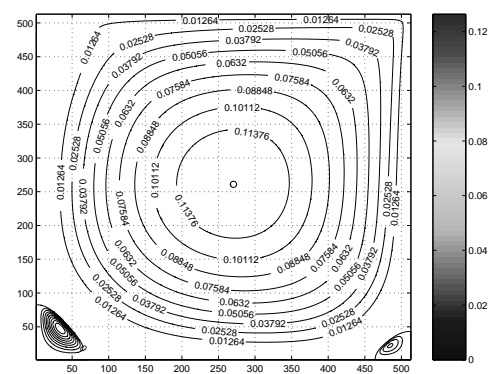

(c) $t=150$

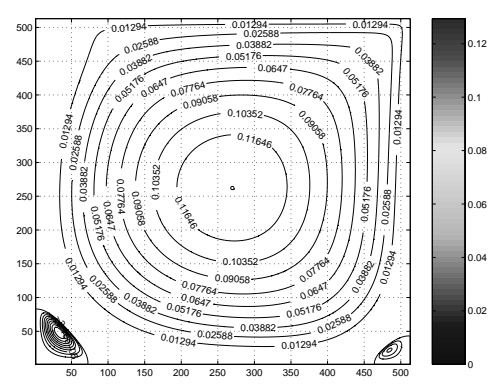

(b) $t=120$



(d) $t=180$

FiguRE 3. Driven cavity for $\nu=1 / 3200$ : streamfunction contours.

TABLE 4.

\begin{tabular}{|c|c|c|c|c|}
\hline time & quantity & $193 \times 193$ & $257 \times 257$ & $513 \times 513$ \\
\hline 60 & $\max |\psi|$ & 0.2007 & 0.1766 & 0.1364 \\
& $(\bar{x}, \bar{y})$ & $(0.5260,0.5156)$ & $(0.5273,0.5156)$ & $(0.5332,0.5098)$ \\
& $\xi(\bar{x}, \bar{y})$ & 4.5183 & 3.8447 & 2.7479 \\
\hline 120 & $\max |\psi|$ & 0.2017 & 0.1734 & 0.1264 \\
& $(\bar{x}, \bar{y})$ & $(0.5260,0.5156)$ & $(0.5273,0.5156)$ & $(0.5254,0.5078)$ \\
& $\xi(\bar{x}, \bar{y})$ & 4.4479 & 3.5662 & 2.1420 \\
\hline 150 & $\max |\psi|$ & 0.2018 & 0.1730 & 0.1252 \\
& $(\bar{x}, \bar{y})$ & $(0.5260,0.5156)$ & $(0.5273,0.5156)$ & $(0.5254,0.5078)$ \\
& $\xi(\bar{x}, \bar{y})$ & 4.4535 & 3.5434 & 2.0736 \\
\hline 180 & $\max |\psi|$ & 0.2019 & 0.1726 & 0.1246 \\
& $(\bar{x}, \bar{y})$ & $(0.5260,0.5156)$ & $(0.5273,0.5156)$ & $(0.5254,0.5078)$ \\
& $\xi(\bar{x}, \bar{y})$ & 4.4563 & 3.5297 & 2.0447 \\
\hline
\end{tabular}

streamfunction and the vorticity contours, respectively, at $t=60,120,150,180$, using a $513 \times 513$ mesh. Note that the transients to steady state are smooth. In Figures $5 \mathrm{a}-\mathrm{b}$ we present velocity components $u(0.5, y)$ and $v(x, 0.5)$ (solid lines) compared with values obtained in [15] (marked by " + "), for $\nu=1 / 3200$ at $t=90,180$, respectively. 


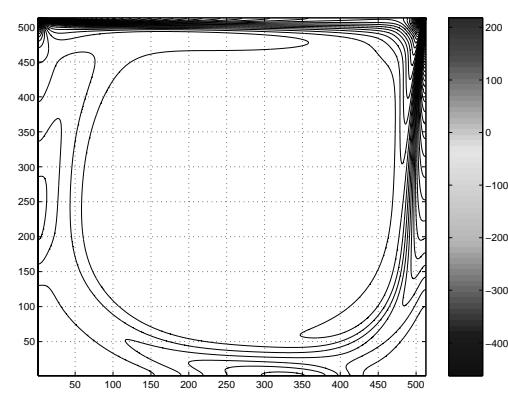

(a) $t=60$



(c) $t=150$

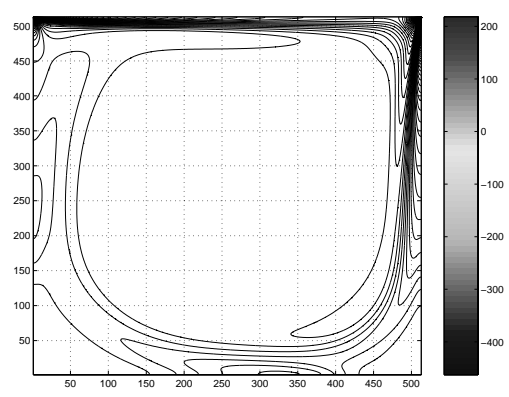

(b) $t=120$

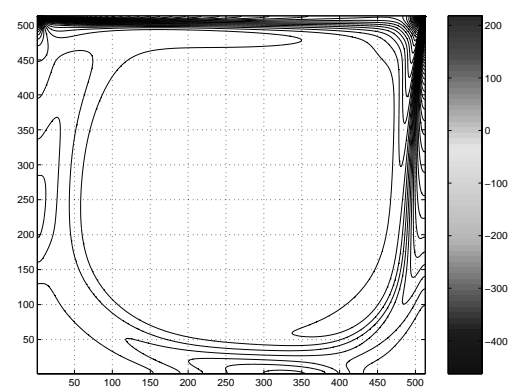

(d) $t=180$

FiguRE 4. Driven cavity for $\nu=1 / 3200$ : vorticity contours.

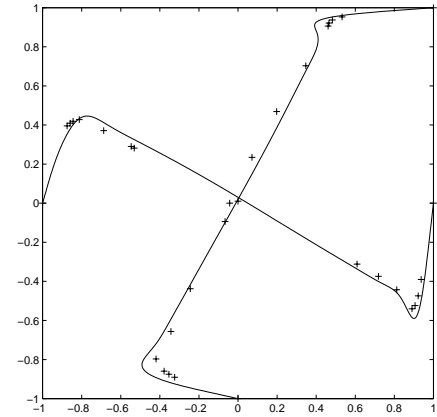

(a) $\nu=1 / 3200, t=90$

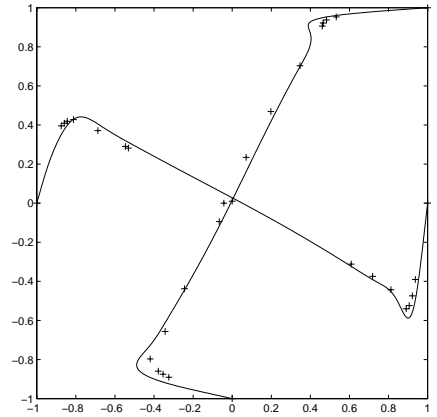

(b) $\nu=1 / 3200, t=180$

FIGURE 5. Velocity components in the driven cavity (solid lines), compared with Ghia et al. (marked "+").

A similar problem, that we considered, has the same geometry $\Omega$, but here the fluid is driven also in the negative $y$-direction at the right-end of $\Omega$. Thus, $u=-1, v=0$ for $y=1, u=0, v=-1$ for $x=1$, $u=v=0$ for $x=0$ and $y=0$. We picked $\nu=1 / 1000$ and a $81 \times 81$ mesh. Figures $6 \mathrm{a}-\mathrm{d}$ and $7 \mathrm{a}-\mathrm{d}$ represent the streamfunction and the vorticity evolution, respectively, at $t=0.04,0.39,3.90$ and 7.81 . 


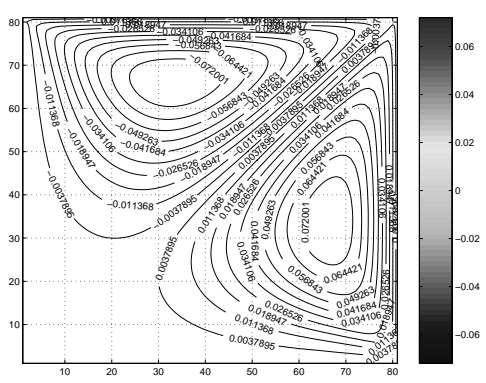

(a) $t=0.04$

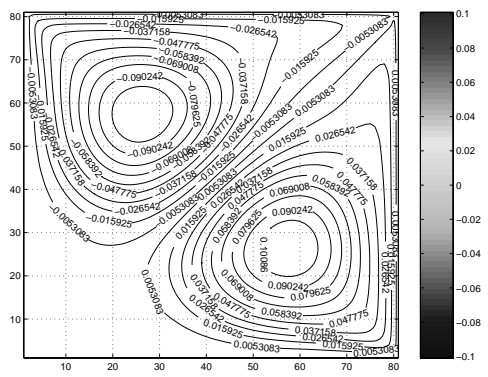

(c) $t=3.90$

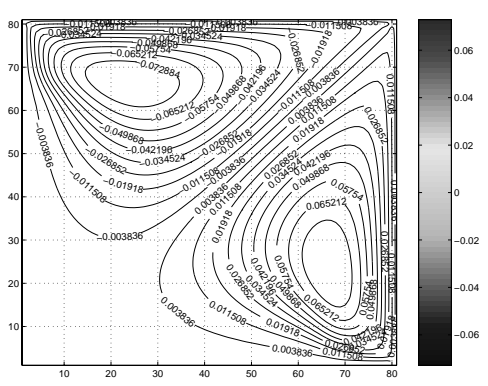

(b) $t=0.39$

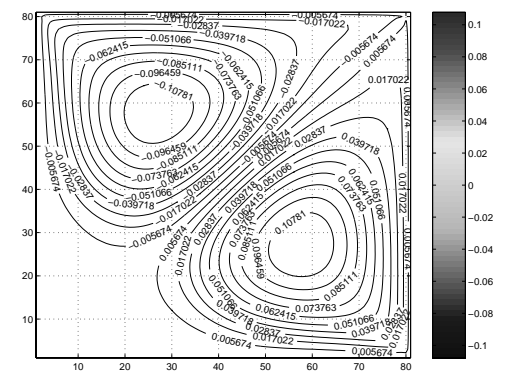

(d) $t=7.81$

Figure 6. Double-driven cavity for $\nu=1 / 1000$ : streamfunction contours.

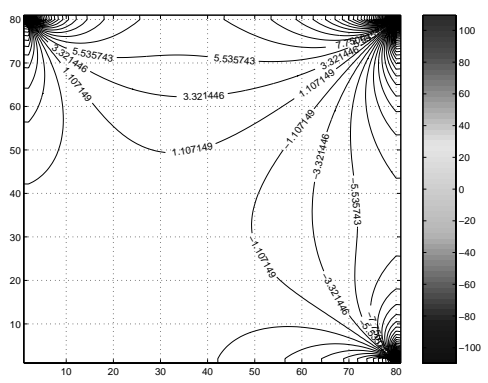

(a) $t=0.04$

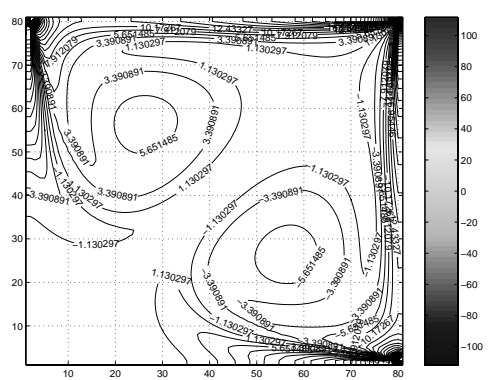

(c) $t=3.90$

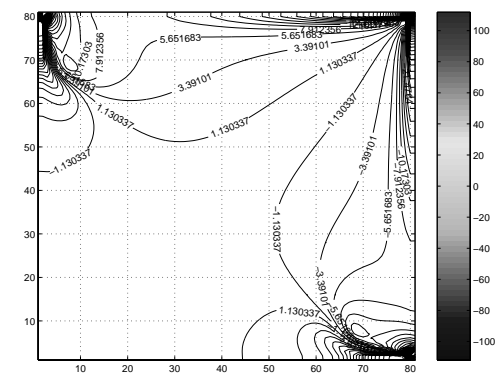

(b) $t=0.39$

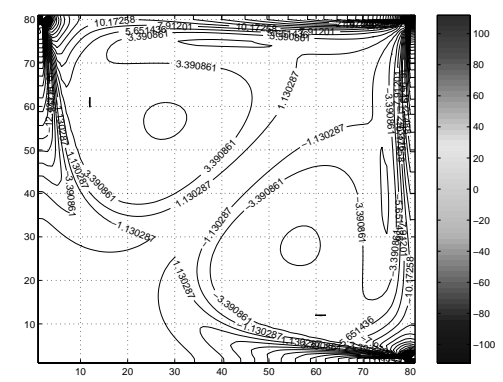

(d) $t=7.81$

FiguRE 7. Double-driven cavity for $\nu=1 / 1000$ : vorticity contours. 




(a) $t=1.5$



(c) $t=18$

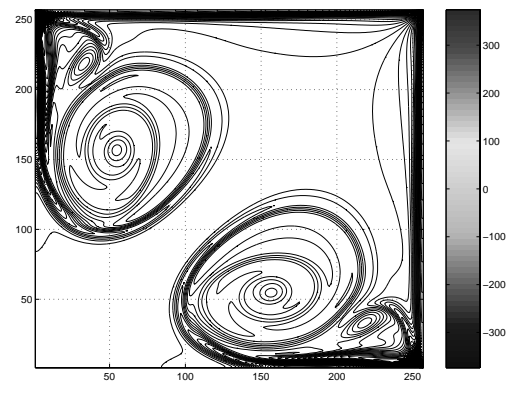

(b) $t=3$

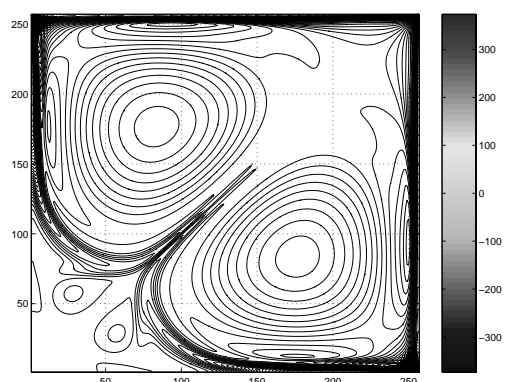

(d) $t=45$

Figure 8. Double-driven cavity for $\nu=1 / 10000$ : vorticity contours.

In Figures 8a-d we display vorticity contours at $t=1.5,3,18,45$ respectively for $\nu=1 / 10000$ with a $257 \times 257$ grid.

Note that the computational results remain stable, retain the symmetry along $y=x$ and capture small scale phenomenon at high Reynolds numbers. In [27] a Hopf bifurcation and break of symmetry are observed at $\nu=1 / 5000$, but we could not confirm it. The differences may be explained by the observation that in [27] the initial condition was taken as the steady-state solution of the same problem with a lower Reynolds number. Here the flow starts impulsively from zero and a steady-state is not yet fully reached.

We further demonstrate results for a flow over a square cylinder. The computational domain is $0 \leq x \leq$ $12,0 \leq y \leq 3$, and the square cylinder lies at $8 \leq x \leq 9,1 \leq y \leq 2$. On the outer boundaries we impose $u=-1, v=0$, and on the inner boundary (the cylinder) $u=v=0$. We picked $\nu=10^{-3}$. Figures 9a-d display the velocity field at $t=2.5,5,7.5,10$ respectively, using a $121 \times 31$ mesh. A Karman vortex street develops as time evolves (see [21] for computations using a control volume formulation, [20] for computations including ground effects and [25] for experimental results).

Our last example is a backward facing step, so that the inflow section is smaller than the outflow one. We thank O. Pironneau for suggesting to us this example, including the initial data. This geometry produces a fluid recirculation zone, that is clearly captured in the results. The setup is shown in Figure 10. We impose a parabolic inflow at the entrance $(x=0)$, i.e., $u=1-y^{2}, v=0$ and a parabolic outflow at the exit $(x=12)$, i.e., $u=C(1-y)(y+2)$. The constant $C$ is determined such that the flux at the entrance equals the flux at the exit. Thus $C=8 / 27$. We picked $\nu=1 / 1000$.

In Figures 11a-d we show contours of the horizontal velocity $u$ at times $1.25,2.5,5,7.5$ on a $121 \times 31$ mesh. Then, for the same times, we show the corresponding contours of the streamfunction in Figures 12a-d, and the corresponding velocity maps in Figures 13a-d. Similar computations were done for the physical domain $0 \leq x \leq 30,-2 \leq y \leq 1$, where the step lies in $0 \leq x \leq 3,-2 \leq y \leq-1$ with $\nu=1 / 150$. Figures $14 \mathrm{a}-\mathrm{d}$ 

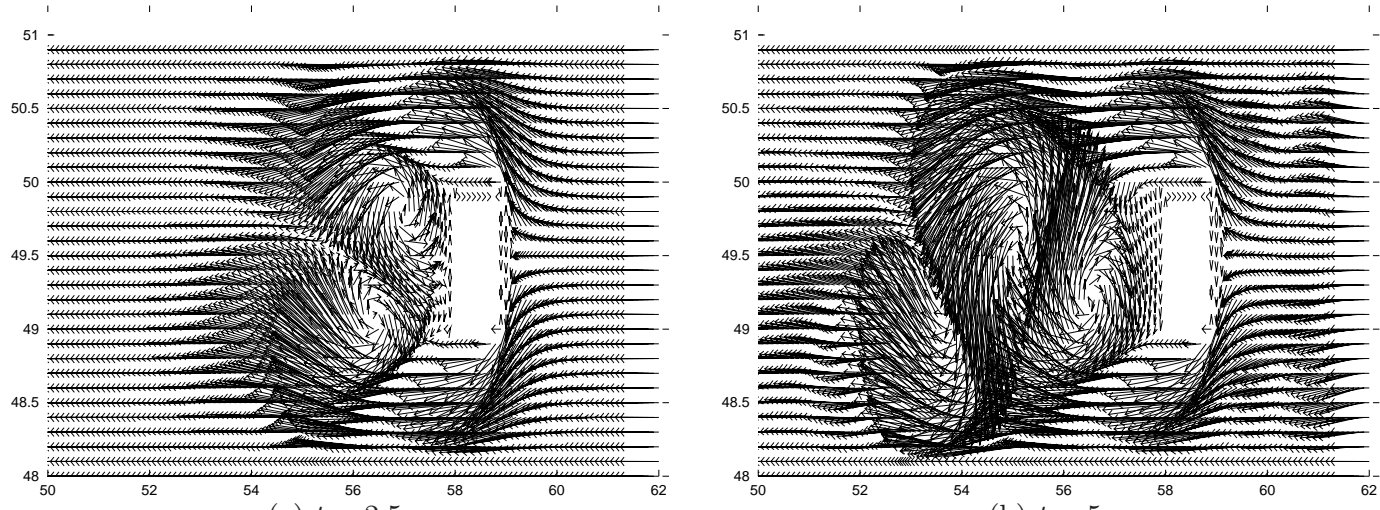

(a) $t=2.5$

(b) $t=5$
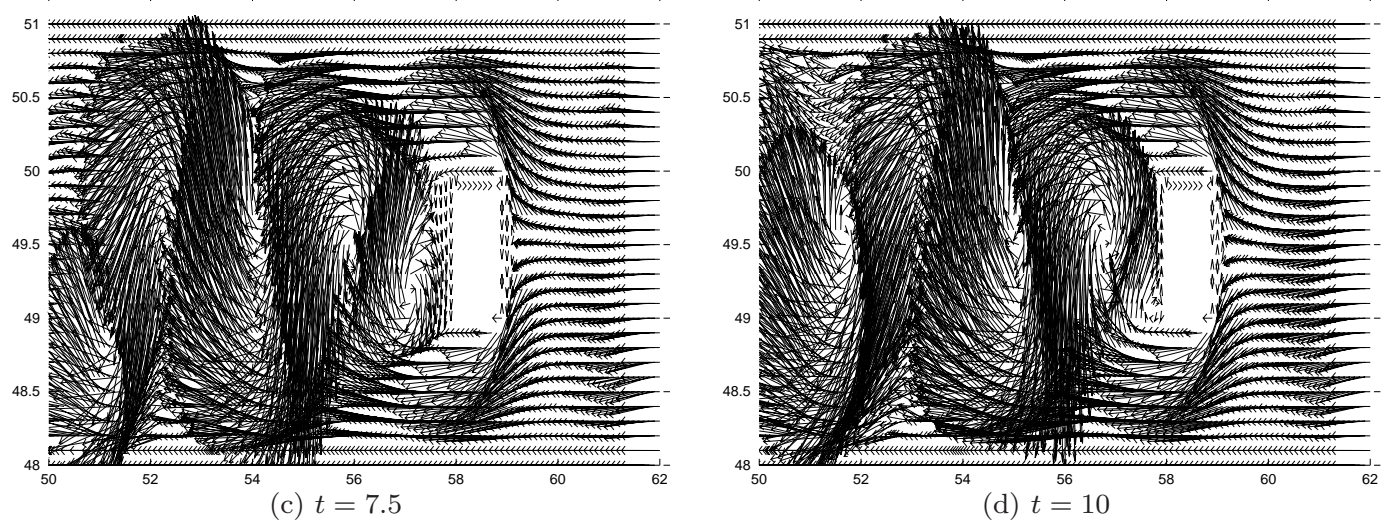

FiguRE 9. Velocity field for a flow over a square cylinder.

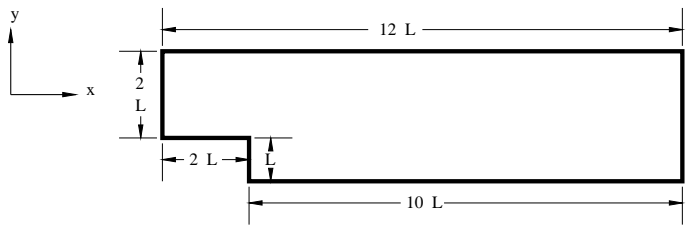

Figure 10. A backward facing step. 


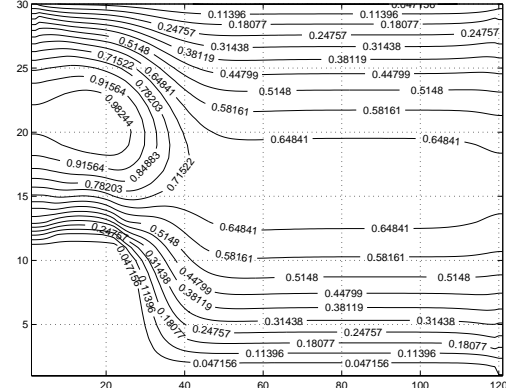

(a) $t=1.25$

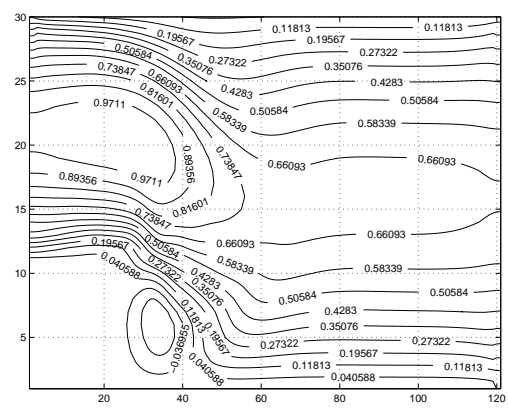

(c) $t=5$

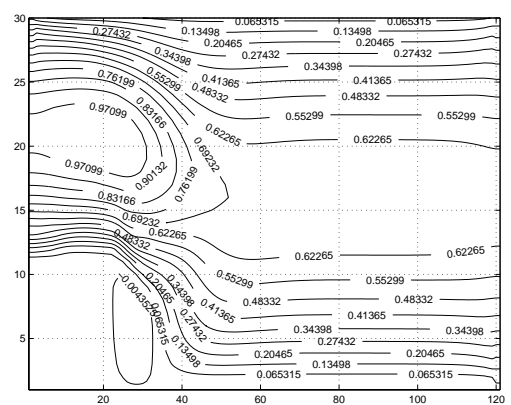

(b) $t=2.5$

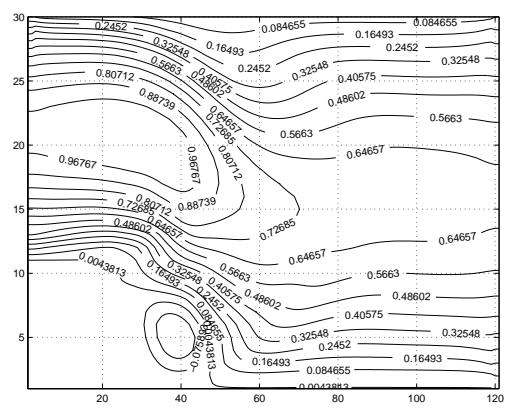

(d) $t=7.5$

FIGURE 11. Horizontal velocity in backward facing step for $\nu=1 / 1000$.



(a) $t=1.25$

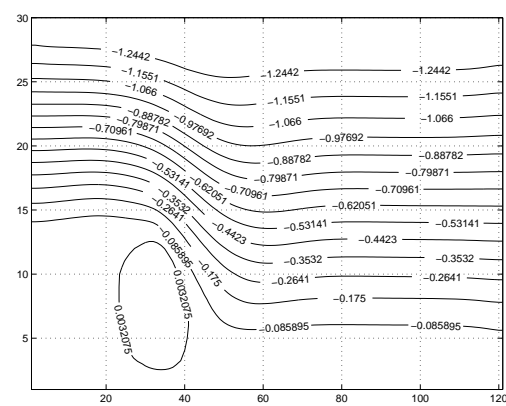

(c) $t=5$

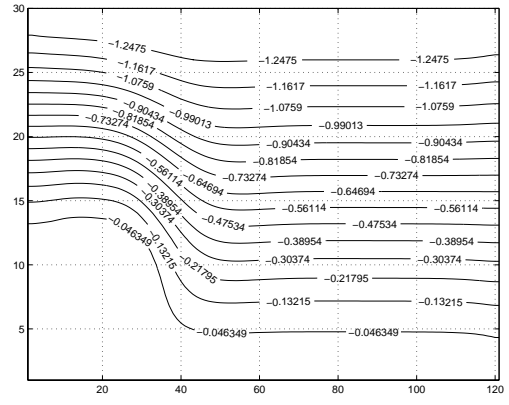

(b) $t=2.5$

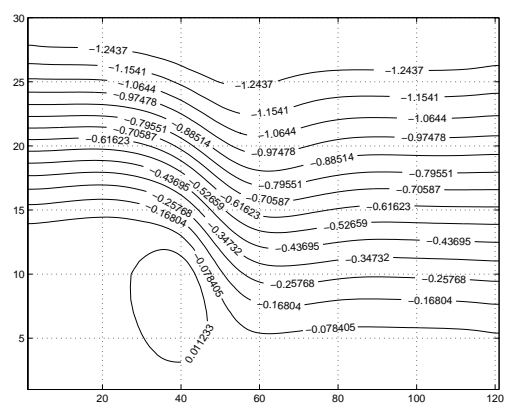

(d) $t=7.5$

FiguRE 12. Streamfunction in backward facing step for $\nu=1 / 1000$. 


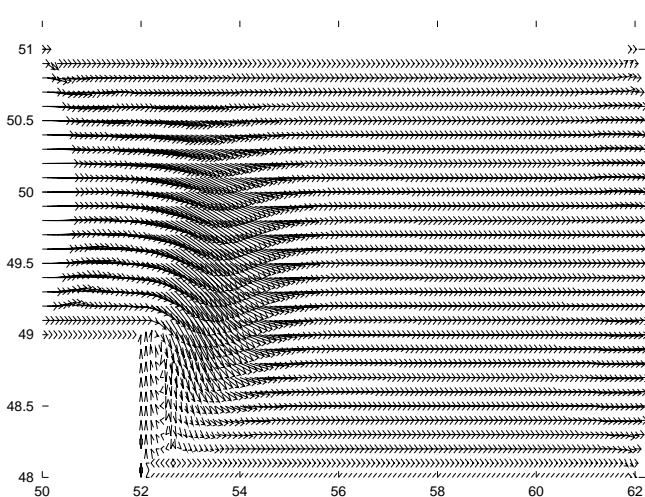

(a) $t=1.25$

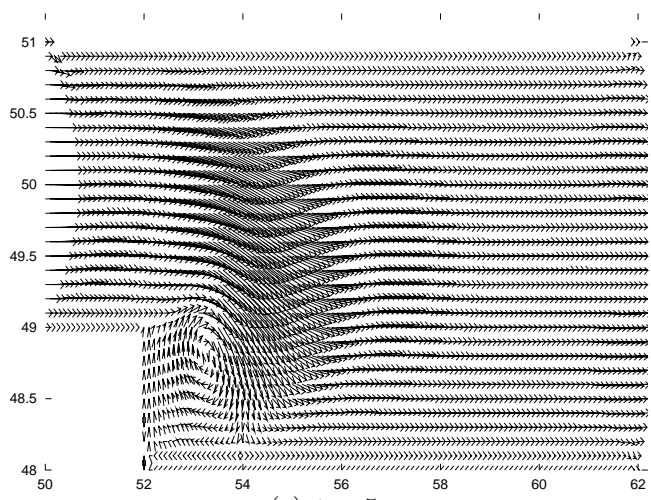

(c) $t=5$

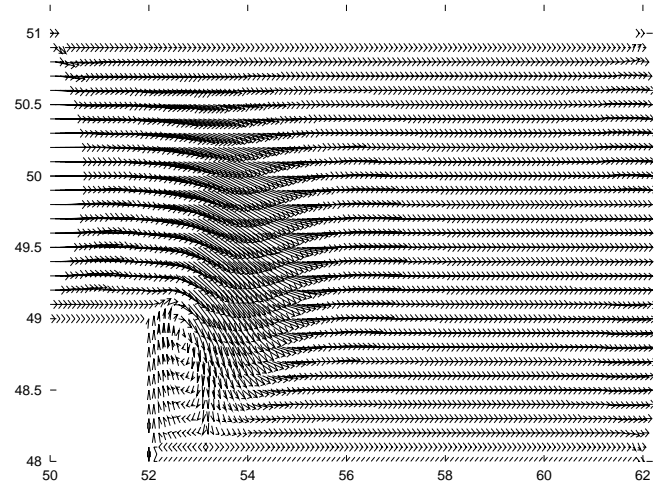

(b) $t=2.5$

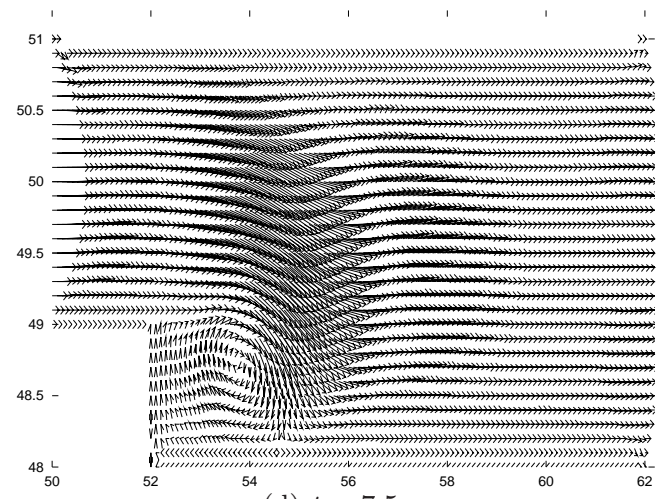

(d) $t=7.5$

FIGURE 13. Velocity field in backward facing step for $\nu=1 / 1000$.

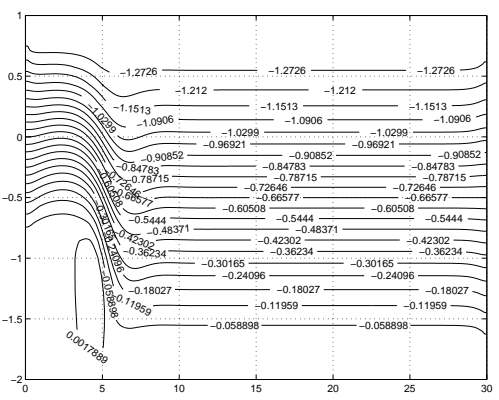

(a) $t=5$



(c) $t=10$

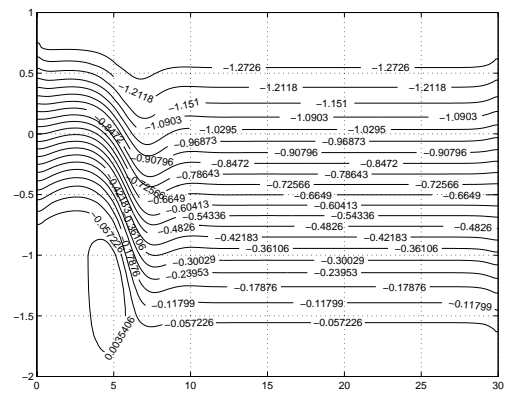

(b) $t=7.5$

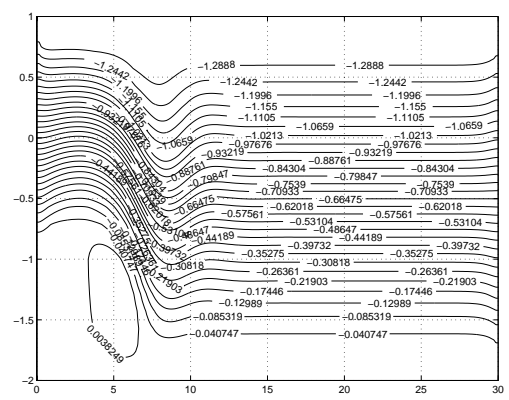

(d) $t=12.5$

Figure 14. Streamfunction contours in backward facing step for $\nu=1 / 150$. 
represent streamfunction contours at $t=5,7.5,10,12.5$ respectively. Note that the length of the wake behind the cylinder is approximately of 8.2 length units. The latter compares favorably with computations done by Glowinski [16].

Acknowledgements. It is a pleasure to thank Professors A. Chorin, R. Glowinski, S. Kamin, O. Pironneau and R. Temam for very useful discussions concerning this paper as well as the broader issues of numerical resolution of the NavierStokes equations. We are grateful to Professor Alexandre Ern for pointing out to us the works of Quartapelle and his collaborators, and to Professors Christophe Besse and Komla Domelevo for pointing out an error in an earlier version of the paper.

\section{REFERENCES}

[1] C.R. Anderson, Vorticity boundary conditions and boundary vorticity generation for two-dimensional viscous incompressible flows. J. Comp. Phys. 80 (1989) 72-97.

[2] J.B. Bell, P. Colella and H.M. Glaz, A second-order projection method for the incompressible navier-stokes equations. J. Comp. Phys. 85 (1989) 257-283.

[3] M. Ben-Artzi, Vorticity dynamics in planar domains. (In preparation).

[4] M. Ben-Artzi, Global solutions of two-dimensional navier-stokes and euler equations. Arch. Rat. Mech. Anal. 128 (1994) 329-358.

[5] P. Bjorstad, Fast numerical solution of the biharmonic dirichlet problem on rectangles. SIAM J. Numer. Anal. 20 (1983) 59-71.

[6] A.J. Chorin, Numerical solution of the navier-stokes equations. Math. Comp. 22 (1968) 745-762.

[7] A.J. Chorin, Vortex sheet approximation of boundary layers. J. Comp. Phys. 27 (1978) 428-442.

[8] A.J. Chorin and J.E. Marsden, A mathematical introduction to fluid mechanics. 2nd edn., Springer-Verlag, New York (1990).

[9] E.J. Dean, R. Glowinski and O. Pironneau, Iterative solution of the stream function-vorticity formulation of the stokes problem, application to the numerical simulation of incompressible viscous flow. Comput. Method Appl. Mech. Engrg. 87 (1991) 117-155.

[10] S.C.R. Dennis and L. Quartapelle, Some uses of green's theorem in solving the navier-stokes equations. Internat. J. Numer. Methods Fluids 9 (1989) 871-890.

[11] W. E and J.-G. Liu, Essentially compact schemes for unsteady viscous incompressible flows. J. Comp. Phys. 126 (1996) $122-138$.

[12] W. E and J.-G. Liu, Vorticity boundary condition and related issues for finite difference scheme. J. Comp. Phys. 124 (1996) $368-382$.

[13] W. E and J.-G. Liu, Finite difference methods for 3-d viscous incompressible flows in the vorticity-vector potential formulation on nonstaggered grids. J. Comp. Phys. 138 (1997) 57-82.

[14] D. Fishelov, Simulation of three-dimensional turbulent flow in non-cartesian geometry. J. Comp. Phys. 115 (1994) $249-266$.

[15] U. Ghia, K.N. Ghia and C.T. Shin, High-re solutions for incompressible flow using the navier-stokes equations and a multigrid method. J. Comp. Phys. 48 (1982) 387-411.

[16] R. Glowinski, Personal communication.

[17] P.M. Gresho, Incompressible fluid dynamics: some fundamental formulation issues. Ann. Rev. Fluid Mech. 23 (1991) $413-453$.

[18] P.M. Gresho and S.T. Chan, On the theory of semi-implicit projection methods for viscous incompressible flow and its implementation via a finite element method that also introduces a nearly consistent mass matrix, parts I-II. Internat. J. Numer. Methods Fluids 11 (1990) 587-659.

[19] K.E. Gustafson and J.A. Sethian (Eds.), Vortex methods and vortex motion. SIAM, Philadelphia (1991).

[20] R.R. Hwang and C-C. Yao, A numerical study of vortex shedding from a square cylinder with ground effect. J. Fluids Eng. 119 (1997) 512-518.

[21] K.M. Kelkar and S.V. Patankar, Numerical prediction of vortex sheddind behind a square cylinder. Internat. J. Numer. Methods Fluids 14 (1992) 327-341.

[22] O.A. Ladyzhenskaya, The mathematical theory of viscous incompressible flow. Gordon and Breach, New York (1963).

[23] L.D. Landau and E.M. Lifshitz, Fluid mechanics, Chap. II, Sec. 15. Pergamon Press, New York (1959).

[24] J. Leray, Etudes de diverses equations integrales non lineaires et des quelques problemes que pose l'hydrodynamique. J. Math. Pures Appl. 12 (1933) 1-82.

[25] D.A. Lyn, S. Einav, S. Rodi and J.H. Park, A laser-doppler velocometry study of ensemble-averaged characteristics of the turbulent near wake of a square cylinder. J. Fluid Mech. 304 (1995) 285-319.

[26] S.A. Orszag and M. Israeli, in Numerical simulation of viscous incompressible flows, M. van Dyke, W.A. Vincenti, J.V. Wehausen, Eds., Ann. Rev. Fluid Mech. 6 (1974) 281-318.

[27] T.W. Pan and R. Glowinski, A projection/wave-like equation method for the numerical simulation of incompressible viscous fluid flow modeled by the navier-stokes equations. Comput. Fluid Dynamics 9 (2000). 
[28] O. Pironneau, Finite element methods for fluids. John Wiley \& Sons, New York (1989).

[29] L. Quartapelle, Numerical solution of the incompressible Navier-Stokes equations. Birkhauser Verlag, Basel (1993).

[30] L. Quartapelle and F. Valz-Gris, Projection conditions on the vorticity in viscous incompressible flows. Internat. J. Numer. Methods Fluids 1 (1981) 129-144.

[31] R. Temam, Sur l'approximation de la solution des équations de Navier-Stokes par la méthode des pas fractionnaires II. Arch. Ration. Mech. Anal. 33 (1969) 377-385.

[32] R. Temam, Navier-Stokes Equations. North-Holland, Amsterdam (1979).

[33] T.E. Tezduyar, J. Liou, D.K. Ganjoo and M. Behr, Solution techniques for the vorticity-streamfunction formulation of the two-dimensional unsteady incompressible flows. Internat. J. Numer. Methods Fluids 11 (1990) 515-539.

To access this journal online:

www.edpsciences.org 